\title{
Caveat Emptor: Let Our Acclaim of the Apotheosis of PRRT Not Blind Us to the Error of Prometheus
}

\author{
Lisa Bodei ${ }^{1}$, Ken Herrmann ${ }^{2,3}$, Richard P. Baum ${ }^{4}$, Mark Kidd ${ }^{5}$, Anna Malczewska ${ }^{6}$, and Irvin M. Modlin ${ }^{7}$ \\ ${ }^{1}$ Memorial Sloan Kettering Cancer Center, New York, New York; ${ }^{2}$ Department of Molecular and Medical Pharmacology, David \\ Geffen School of Medicine at UCLA, Los Angeles, California; ${ }^{3}$ Klinik für Nuklearmedizin, Universitätsklinikum Essen, Essen, \\ Germany; ${ }^{4}$ Theranostics Center for Molecular Radiotherapy and Imaging, Zentralklinik Bad Berka, Bad Berka, Germany; ${ }^{5}$ Wren \\ Laboratories, Branford, Connecticut; ${ }^{6}$ Department of Endocrinology and Neuroendocrine Tumors, Medical University of Silesia, \\ Katowice, Poland; and ${ }^{7}$ Yale School of Medicine, Department of Surgery, New Haven, Connecticut
}

$\mathbf{M}$ odern medicine has made extraordinary advances as various scientific technologies are applied to amplify and diversify the diagnostic and therapeutic strategies that physicians offer. Inherent in the natural enthusiasm that accompanies these developments is an exuberance and sometimes even hubris. The familiar jargon of molecular genomics, precision medicine, and targeted therapy and the current milieu of prognostics, predictors, and pipelines have dulled our appreciation to the value of common sense. A good example is the extraordinary advances in the radioisotopic treatment of disease that have been made since Saul Hertz (19051950) first initiated the concept of theranostics using radioactive iodine to treat thyroid disease. Since then, the number of isotopes has proliferated, the types of cancers treated have diversified, and the targeting strategies have burgeoned. With this diffuse propagation, it has become apparent that it is timely to implement simple and thoughtful strategies to obviate overuse in disease groups not judiciously stratified for benefit and risk.

It is generally accepted that peptide receptor radionuclide therapy (PRRT) is an efficient and well-tolerated treatment for well-differentiated neuroendocrine tumors (NETs) of the gastroenteropancreatic and bronchopulmonary tract that overexpress somatostatin receptors (SSRs) (1). More recently, the significant benefit of PRRT versus conventional therapies was demonstrated (2) and the therapy ratified by the Food and Drug Administration, European Medicines Agency, and National Institute for Health and Care Excellence. Indeed, this initiated a paradigm shift in NET treatment.

PRRT is undertaken in patients with progressive gastroenteropancreatic or bronchopulmonary NETs on the basis of positive ${ }^{68}$ Ga-DOTA-peptide PET/CT (DOTATATE/DOTATOC) or scintigraphic imaging (1). The expectation is that very few patients will experience adverse events and that most will benefit (1). There are, however, few objective criteria to predict therapeutic benefit or quantify toxicity risk (1).

\footnotetext{
Received Oct. 16, 2018; revision accepted Nov. 14, 2018.

For correspondence or reprints contact: Lisa Bodei, Molecular Imaging and Therapy Service, Department of Radiology, Memorial Sloan Kettering Cancer Center, 1275 York Ave., Box 77, New York, NY 10065.

E-mail: bodeil@mskcc.org

COPYRIGHT (c) 2019 by the Society of Nuclear Medicine and Molecular Imaging. DOI: 10.2967/jnumed.118.219386
}

The only current predictive assessment for treatment efficacy of PRRT is the intensity of neoplastic SSR expression, namely tumor uptake, at pretherapeutic SSR imaging. This is represented as either visual scoring by conventional OctreoScan or as an SUV measurement at ${ }^{68} \mathrm{Ga}$-DOTA-peptide PET/CT. The level of SSR expression represents an estimate of therapeutic targeting and has been proposed as both a predictive and a prognostic factor. As might be envisaged, this is suboptimal because only $60 \%$ of individuals with elevated uptake (grade 4 on the Krenning scale, a visual assessment scoring system) demonstrate minor to complete remissions (1).

Other factors including tumor grade, glucose metabolism, performance status, and disease burden have been assessed but function as prognosticators; their value is independent of any therapeutic intervention. Thus, ${ }^{18} \mathrm{~F}-\mathrm{FDG}$ avidity, although associated with differences in progression-free survival, reflects a prognostic rather than PRRT-predictive value (3). Similarly, low-grade tumors respond for longer than high-grade tumors to PRRT. This too reflects that grade is prognostic. Both performance status and extent of disease are also prognostic as expected (1). It is well recognized in biologic science that the presence of a target cannot objectively predetermine the level of agent targeting; certainly, it cannot predict the full effect thereof.

Given these caveats, it is currently not feasible in a specific individual to objectively predict the effect of PRRT. In other therapeutic environments such as antibiotic administration or breast or lung cancer therapy, the prediction of therapeutic efficacy is considered a sine qua non. Given the fact that PRRT involves radionuclide exposure, potential adverse events, and cost, prediction of efficacy is a critical health-care consideration. It captures 2 issues: namely the clinical cost-benefit ratio of the therapy and optimal stratification of patients. The latter is critical because some may require additional therapy of a different type to optimize outcome goals.

We propose that special consideration should be given to developing rigorous scientific strategies to predict PRRT efficacy. Otherwise, it is foreseeable, given the anticipated global expansion and current enthusiasm for PRRT, that some patients may not benefit from this therapy, with a consequent decline in interest by the referring oncologists. It would seem prudent to direct PRRT to those patients who are most likely to respond. A tool that provides appropriate patient stratification should thus be developed and tested.

Stratification is needed, because of the well-recognized interpatient and intrapatient differences, tumor heterogeneity, evolving 
tumor biology over time, and varying tumor characteristics in metastatic disease. There is little doubt that SSR scintigraphy and PET/CT are formidable instruments. However, difficulties in imaging tumor heterogeneity and the intrinsic limits of system resolution identify that ${ }^{68} \mathrm{Ga}$-DOTA-peptide evaluation alone cannot predict the complex biologic phenomena of radiation response. It is therefore quixotic to accept that the current tools used for prediction are effective. New molecular tools and a revised management strategy are required.

The search for a patient-specific multigene genomic signature that can predict tumor response in a single individual is a major field of exploration in several cancers. Multiple single and multianalyte biomarkers are commonly used to select patients for treatment, in terms of a druggable target or altered pathway. Examples include measuring human epidermal growth factor receptor 2 expression in vivo with PET/CT using ${ }^{89} \mathrm{Zr}$-labeled pertuzumab (4) (which can be regarded as similar to SSR expression for NETs) or the measurement of tissue messenger RNAbased multigene signatures, such as MammaPrint (70 genes) (5), which identifies favorable subpopulations of hormone-sensitive breast cancer. Recent results from the phase III TAILORx trial demonstrated that treatment response prediction with a tumorderived multigene signature was clinically effective (6).

Studies of circulating NET-specific transcripts in patients undergoing ${ }^{177} \mathrm{Lu}$-DOTATATE therapy have demonstrated that pretreatment blood levels accurately predicted response-tumor control-by PRRT (7). Circulating genes regulating growth factor signaling (mainly the RAF family) and genes involved in metabolism (e.g., PANK2) effectively identified tumors responsive to PRRT. These observations constitute the basis for the development of a PRRT-specific predictor test, which combines the genes involved in growth factor expression/metabolism with tissue grade (Ki-67). This genomic biomarker was validated in 3 independent center cohorts treated with ${ }^{177} \mathrm{Lu}-\mathrm{PRRT}$ and demonstrated a $95 \%$ accuracy in predicting the response to PRRT (8). As importantly, this molecular tool was not effective for predicting response to cold octreotide and did not function as a prognostic marker. It is therefore a specific PRRT-prediction tool.

As a discipline, nuclear medicine must learn the lessons learnt from treatment of other cancers and integrate such knowledge into the emerging world of PRRT. A patient-specific predictive test for PRRT with a predictive value of $95 \%$ is already available (8). It is likely that the integration of genomic information with ${ }^{68} \mathrm{Ga}-$ DOTA-peptide SUV will further delineate individuals who will benefit most from treatment or for whom alternative therapeutic strategies should be considered, including the use of additional agents, such as combinations, to overcome the likely radioresistance. Alternatively, given the expense and the known (albeit usually modest) adverse events, demonstration of a likely negative outcome would both be fiscally prudent medicine and avoid unnecessary radiation exposure. Proof of principle has been demonstrated (8).

There is no need to exhibit temerity in the prediction of the outcome of PRRT; it is simply a question of optimizing therapy delivery. The potential impact of PRRT is likely to be so substantial that it should be deployed carefully to ensure we avoid ineffective or suboptimal outcomes.

Prediction of the efficacy of a therapy has been a fundamental criterion for successful disease management since the introduction of antibiotics. The lack of stratification and the consequent suboptimal outcomes, compared with the treatment potential, raises the specter that a long-ostracized therapy (PRRT) might become labeled as a caveat emptor-type therapy. After an initial enthusiasm, such an outcome could once again relegate PRRT to a minor role or to a last resort approach in the treatment paradigm. We should be mindful of the fate of Prometheus who, in seeking to do good for humanity, incurred the wrath of the divine leaders. Let us thoughtfully define and predict the application of PRRT to ensure its maximal benefit to our patients.

\section{DISCLOSURE}

Lisa Bodei is a consultant to Ipsen and AAA. Ken Herrmann is a consultant for Sofie Biosciences. Mark Kidd is an employee of Wren Laboratories. Irvin M. Modlin is a consultant to Wren Laboratories. No other potential conflict of interest relevant to this article was reported.

\section{REFERENCES}

1. Kwekkeboom DJ, Kam BL, van Essen M, et al. Somatostatin-receptor-based imaging and therapy of gastroenteropancreatic neuroendocrine tumors. Endocr Relat Cancer. 2010;17:R53-R73.

2. Strosberg J, El-Haddad G, Wolin E, et al. Phase 3 trial of ${ }^{177} \mathrm{Lu}$-Dotatate for midgut neuroendocrine tumors. N Engl J Med. 2017;376:125-135.

3. Sansovini M, Severi S, Ianniello A, et al. Long-term follow-up and role of FDG PET in advanced pancreatic neuroendocrine patients treated with ${ }^{177} \mathrm{Lu}-\mathrm{D}$ OTATATE. Eur J Nucl Med Mol Imaging. 2017;44:490-499.

4. Ulaner GA, Lyashchenko SK, Riedl C, et al. First-in-human human epidermal growth factor receptor 2-targeted imaging using ${ }^{89} \mathrm{Zr}$-pertuzumab PET/CT: dosimetry and clinical application in patients with breast cancer. $\mathrm{J} \mathrm{Nucl} \mathrm{Med}$. 2018;59:900-906.

5. Ward S, Scope A, Rafia R, et al. Gene expression profiling and expanded immunohistochemistry tests to guide the use of adjuvant chemotherapy in breast cancer management: a systematic review and cost-effectiveness analysis. Health Technol Assess. 2013;17:1-302.

6. Sparano JA, Gray RJ, Makower DF, et al. Adjuvant chemotherapy guided by a 21-gene expression assay in breast cancer. N Engl J Med. 2018;379:111-121.

7. Bodei L, Kidd M, Modlin IM, et al. Measurement of circulating transcripts and gene cluster analysis predicts and defines therapeutic efficacy of peptide receptor radionuclide therapy (PRRT) in neuroendocrine tumors. Eur J Nucl Med Mol Imaging. 2016;43:839-851.

8. Bodei L, Kidd MS, Singh A, et al. PRRT genomic signature in blood for prediction of ${ }^{177}$ Lu-octreotate efficacy. Eur J Nucl Med Mol Imaging. 2018;45:11551169. 